\title{
RASHES AND VASCULITIS
}

\author{
R A Watts, D G I Scott
}

\section{Differential diagnosis of rash} and arthritis

- Infection

- Drug reaction

- Sarcoidosis

- Juvenile chronic arthritis

- Connective tissue disease

- Psoriasis

- Vasculitis

\section{Infection}

\section{Common infectious causes of rash and arthritis}

Viral exanthemata

Parvovirus B19

Rubella

Hepatitis B

Bacteria

Neisseria gonorrhoeae

Neisseria meningitidis

Streptococci

Spirochaetes

Borrelia burgdorferi

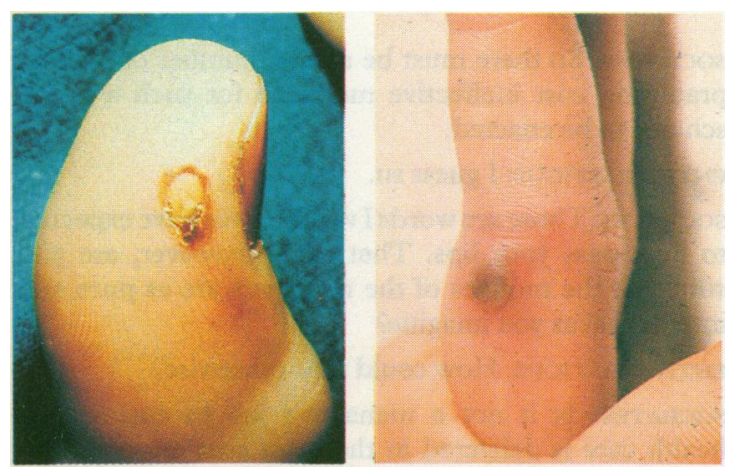

Gonococcal pustules in disseminated infection with Neisseria gonorrhoeae.

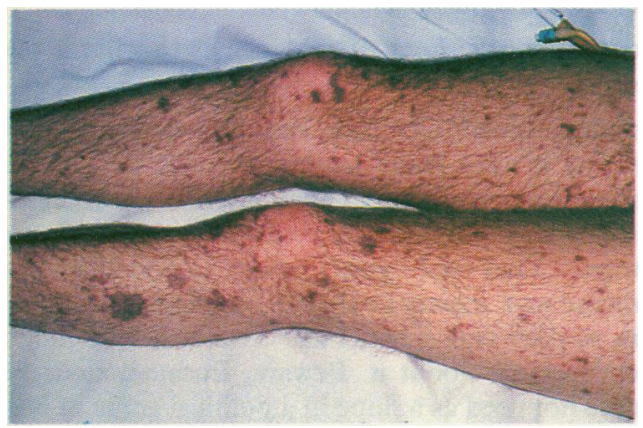

Haemorrhagic pustular rash in disseminated infection with Neisseria meningitidis.
Many patients with rheumatological disorders either present with a rash or develop a rash in the course of the disease. This is particularly true of the connective tissue diseases and psoriasis, which have been described elsewhere in this series. The differential diagnosis of rash and arthritis is wide, but in most cases a diagnosis can be made on the basis of history, clinical examination, and appropriate blood tests.

Several microorganisms can cause both a rash and arthritis, either by direct infection or by immune mediated mechanisms.

\section{Streptococcal infections}

Rheumatic fever is caused by an immune mediated response to group A $\beta$ haemolytic streptococcal pharyngitis. It is rare in the developed world, but recent outbreaks have been reported in the United States and Europe. Most cases occur in people aged 5-16. The characteristic skin lesion is erythema marginatum. The arthritis affects large joints and migrates from joint to joint, each joint being affected for only two to three days, and lasts overall for three weeks. Nodules may develop over bony prominences; other features include chorea and carditis. Infections are treated with penicillin and anti-inflammatory drugs.

A reactive arthritis can occur after infections with group $A$ and possibly group $\mathrm{G}$ streptococci. The arthritis is more prolonged, lasting for two to three months, and does not migrate between joints. It may be accompanied by a vasculitic rash.

\section{Neisserial infections}

Disseminated infection with Neisseria gonorrhoea is three to five times more common in women than men. Urethritis or cervicitis is often asymptomatic. There is an initial bacteraemic phase with a migratory polyarthritis and typical skin lesions that may be maculopapular, vesicular, or pustular. Tenosynovitis of the hand, wrist, or ankle is common in established disease. $N$ gonorrhoeae is the most common cause of a bacterial arthritis in adults (typically aged 15-30). The organism is usually isolated from a pustule or the genitourinary tract but can be isolated from blood or synovial fluid. It is treated with penicillin.

Infection with $N$ meningitidis is associated with a widespread haemorrhagic pustular rash, particularly over the buttocks and legs, and can rapidly become life threatening. Diagnosis is confirmed by blood culture, and it is treated with penicillin. A self limiting polyarthritis may develop three to five days after the rash, which is not influenced by antibiotic treatment. 


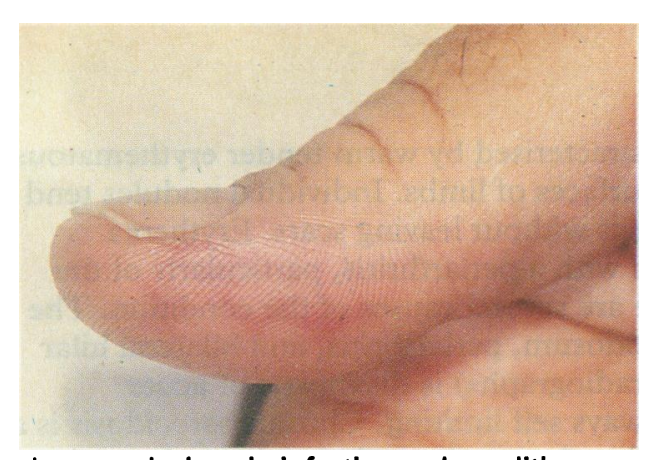

Janeway lesions in infective endocarditis.

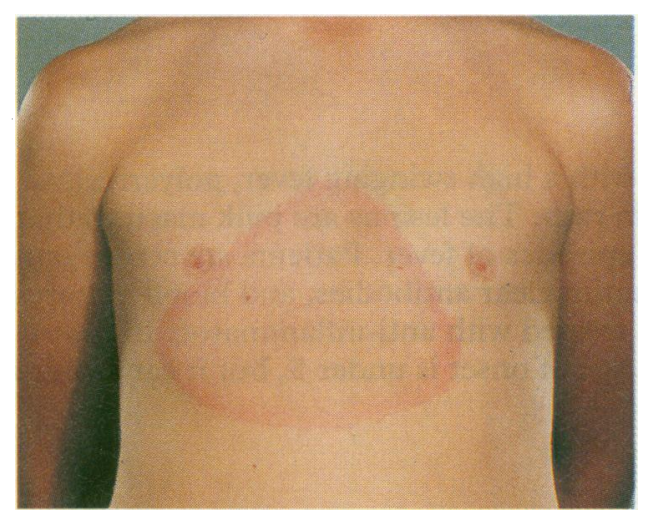

Erythema chronicum migrans at site of tick bite in Lyme disease.

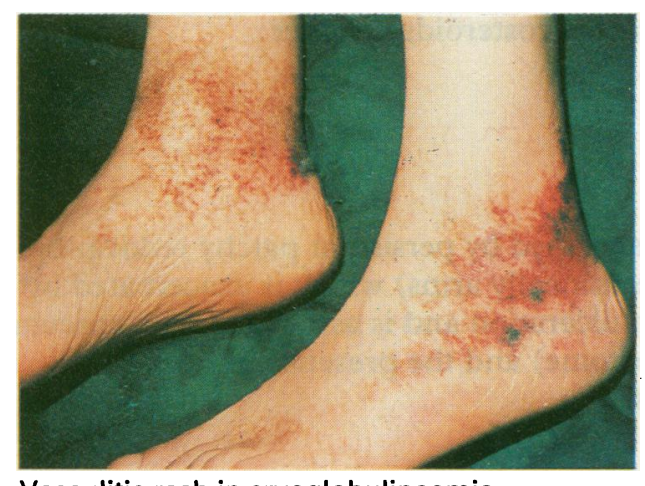

Vasculitic rash in cryoglobulinaemia.

\section{Drugs}

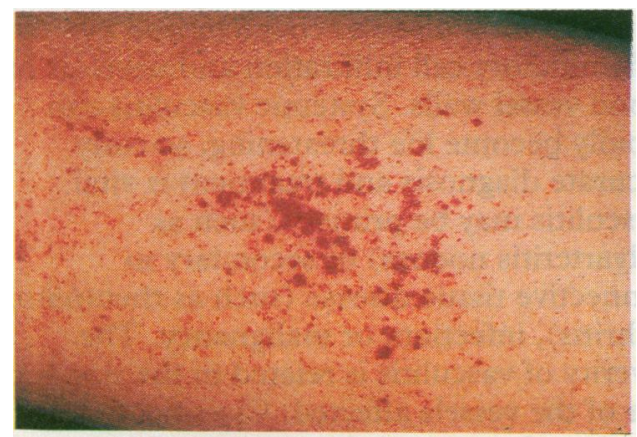

Maculopapular eruption caused by reaction to sulphasalazine (Salazopyrin).

\section{Infective endocarditis}

Several organisms-including streptococci, staphylococci, gram negative bacilli, and coxiella - can cause endocarditis. Polyarthritis may be accompanied by splinter haemorrhages, Janeway lesions (red macules on the thenar and hypothenar eminences), Osler's nodes (tender papules over the extremities of fingers and toes), and clubbing. Diagnosis is made by blood culture and echocardiography.

\section{Lyme disease}

Lyme disease is caused by the spirochaete Borrelia burgdorferi and is transmitted by a bite from an ixodid tick. The earliest feature is the typical rash, erythema chronicum migrans, which occurs at the site of the bite. Patients are often asymptomatic at this stage. The lesion begins as a red macule or papule that expands to form an annular erythematous lesion with an indurated centre. Secondary lesions may develop. Within a few weeks of onset a migratory polyarthritis may develop; in a few patients this becomes a chronic intermittent large joint arthropathy. Other systemic features include meningitis, encephalitis, radiculopathy, and cardiac conduction defects. Diagnosis is made by a serological test for borrelia antibodies. Early disease is treated with tetracycline, while disseminated disease should be treated with a cephalosporin or penicillin.

\section{Rubella}

Rubella virus causes a mild acute illness with a characteristic maculopapular rash and lymphadenopathy that are typically postauricular. A self limiting symmetrical polyarthritis may follow, especially in young women. Chronic joint disease does not occur.

\section{Parvovirus}

Parvovirus B19 is the cause of fifth disease or erythema infectiosum in childhood. The characteristic skin lesion is a "slapped cheek" erythema, which may be associated with a reticular rash over the trunk. An acute self limiting symmetrical polyarthritis occurs, typically in young adult women, and can last for up to one year. A rise in IgM titre is diagnostic, and autoantibodies may be present transiently.

\section{Other infections}

Hepatitis B infection can present with a small vessel vasculitis during the acute illness. Chronic hepatitis B antigenaemia may be associated with classic polyarteritis nodosa. Mixed cryoglobulinaemia may also occur after infection with hepatitis A, B, or C, and patients may present with a vasculitic rash. HIV infection is associated with an increased occurrence of Reiter's syndrome, psoriatic arthritis, and vasculitis that may affect small and medium vessels.

Skin eruptions are a common complication of many drugs, including those used to treat arthritis. They are often mild but may be life threatening.

Non-steroidal anti-inflammatory drugs can be associated with mild maculopapular reactions at any stage of treatment. Phototoxicity is now rare (it was particularly associated with Opren).

Penicillamine can cause pruritus and maculopapular rashes early in treatment, while lichenoid or bullous lesions occur later. It can also induce a lupus-like illness.

Gold treatment-About $15 \%$ of patients treated with gold develop a rash, usually a non-specific maculopapular scaling eruption. Severe erythroderma is rare but can be life threatening.

Sulphasalazine - The sulphonamide part of this drug can cause a maculopapular rash and occasionally toxic epidermal necrolysis.

Immunisation with influenza or rubella vaccine or tetanus toxoid can induce a polyarthritis, which is usually transient. Chronic arthritis has been reported after immunisation, but the exact relation between the arthritis and immunisation is unclear. 


\section{Common causes of erythema nodosum}

- Acute sarcoidosis

- Drugs (such as oral contraceptives, sulphonamides)

- $\beta$ haemolytic streptococci

- Ulcerative colitis

- Crohn's disease

- Tuberculosis

\section{Erythema nodosum}

Erythema nodosum is characterised by warm tender erythematous nodules over the extensor surfaces of limbs. Individual nodules tend to resolve over three to six weeks without leaving scars. Erythema nodosum may be associated with a periarthritis, particularly of the ankles and wrists, and there are several causes of the condition. The combination of erythema nodosum, periarthritis, and bilateral hilar lymphadenopathy (seen in radiographs) is diagnostic of acute sarcoidosis and is almost always self limiting. Chronic sarcoidosis is a multisystem disease in which a destructive arthropathy and papular infiltrative skin lesions may develop. Serum concentrations of angiotensin converting enzyme are often raised in active systemic sarcoidosis.

\section{Still's disease}

This is a systemic illness with a high swinging fever, polyarthritis, and a typical evanescent skin rash. The lesions are pink macules that are most prominent during episodes of fever. Patients are seronegative for rheumatoid factor and antinuclear antibodies, and blood cultures are sterile. The condition is treated with anti-inflammatory drugs or corticosteroids. The typical age of onset is under 5, but it can occur in adults.

\section{Acute febrile neutrophilic dermatosis (Sweet's syndrome)}

This condition occurs in young women and comprises four main features: fever, painful red skin plaques, leucocytosis and a dermal infiltrate with neutrophils. It is associated with myelodysplasia and leukaemia. It is treated with corticosteroids or other immunosuppressive agents.

\section{Livedo reticularis}

Livedo reticularis is characterised by persistent patchy reddish-blue mottling of the legs (and occasionally arms) which is exacerbated by cold weather. It may lead to ulceration and is associated with vascular thrombosis (Sneddon's syndrome) and the presence of antiphospholipid antibodies.

\section{Vasculitis}

\section{Classification of vasculitis \\ Vessels affected Primary \\ Large arteries Giant cell arteritis \\ Takayasu's arteritis \\ Medium arteries Classic polyarteritis nodosa \\ Kawasaki's disease \\ Medium arteries Wegener's and small granulomatosis* \\ vessels \\ Small vessels \\ (leucocyto- \\ clastic) \\ Churg-Strauss syndrome* Microscopic polyangiitis* \\ Secondary \\ Aortitis associated with rheumatoid arthritis Infection (syphilis) Infection (such as hepatitis B) \\ Rheumatoid arthritis, systemic lupus erythematosus, Sjögren's syndrome Drugs \\ Infection (HIV) \\ Henoch-Schönlein purpura Drugs (such as sulphonamides, Essential mixed cryoglobulinaemia \\ penicillins, thiazide diure$$
\begin{gathered}
\text { etc) } \\
\text { Infection }
\end{gathered}
$$

*Diseases commonly associated with antineutrophil cytoplasmic antibodies (antimyeloperoxidase and antiproteinase 3 antibodies) and a substantial risk of renal impairment and which are most responsive to immunosuppression with cyclophosphamide.

The vasculitides are a heterogeneous group of uncommon diseases characterised by inflammatory cell infiltration and necrosis of blood vessel walls. Systemic vasculitis can rapidly become life threatening, so early accurate diagnosis and treatment is vital. Vasculitis may be primary (such as polyarteritis nodosa) or secondary to connective tissue disease (such as rheumatoid arthritis), infection, or malignancy. The severity of vasculitis is related to the size and site of the vessels affected. Classification is also based on vessel size, which reflects treatment strategies. 


\section{Symptoms suggestive of systemic vasculitis}

Systemic-Malaise, fever, weight loss, myalgia, arthralgia

Skin-Purpura (palpable), ulceration, infarction

Ear, nose, and throat-Epistaxis, crusting, sinusitis, deafness

Respiratory-Cough, wheeze, haemoptysis, dyspnoea

Cardiac-Chest pain

GastrointestinaL-Mouth ulcers, abdominal pain, diarrhoea

Neurological-Sensory or motor impairment
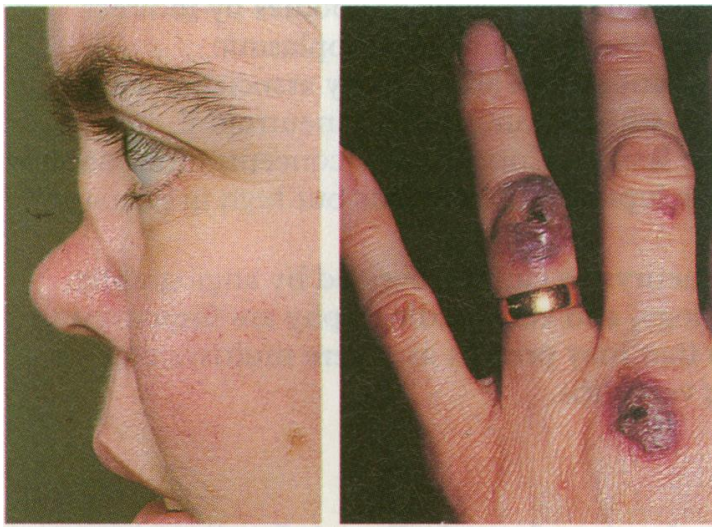

Wegener's granulomatosis: (left) typical saddle nose deformity (reproduced with patient's permission); (right) vasculitic rash.

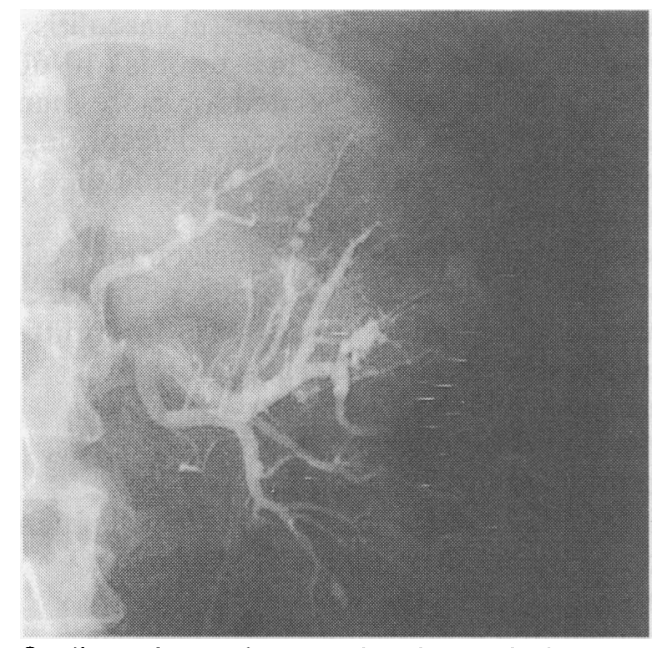

Coeliac axis arteriogram showing typical aneurysm in polyarteritis nodosa.

\section{Systemic necrotising arteritis}

This group of conditions includes the major necrotising vasculitides: classic polyarteritis nodosa, microscopic polyangiitis (also called microscopic polyarteritis nodosa), Wegener's granulomatosis, and Churg-Strauss syndrome, with necrosis of medium and small arteries. They may occur at any age and are not uncommon in elderly people. Primary systemic vasculitis is slightly more common in men. The annual incidence is about 30 cases per million people. The symptoms of vasculitis depend on the size and site of vessel affected and on the individual condition.

Wegener's granulomatosis is characterised by a granulomatous vasculitis of the upper and lower respiratory tracts and glomerulonephritis, but almost any organ system can be affected. The lungs are affected in $45 \%$ of patients at diagnosis. Symptoms of the ear, nose, and throat (such as epistaxis, crusting, and deafness) are particularly associated with this condition and should be sought in all patients with suspected vasculitis. Patients with limited Wegener's granulomatosis - disease without renal impairment-may have a better prognosis. Biopsy of affected organs shows a necrotising arteritis, often with formation of granulomas. Microscopic polyangiitis is a vasculitis of medium arteries and small vessels and mainly affects the kidneys.

Churg-Strauss syndrome is characterised by atopy (in particular late onset asthma), pulmonary involvement ( $75 \%$ of patients have radiographic evidence of infiltration), and eosinophilia. These features can develop several years before the start of systemic disease. Cardiac involvement is a particular feature of Churg-Strauss syndrome and is a determinant of prognosis.

Polyarteritis nodosa is a multisystem vasculitis characterised by formation of aneurysms in medium sized arteries. Patients present with a constitutional illness, often associated with a rash; mononeuritis multiplex, or vascular hypertension. Polyarteritis nodosa may be confined to the skin. Angiography shows the presence of typical aneurysms. Classic polyarteritis nodosa has been associated with chronic hepatitis B antigenaemia, especially in the United States, but this is rare in the United Kingdom.

Kawasaki disease (mucocutaneous lymph node syndrome) is an acute vasculitis that primarily affects infants and young children. It presents as fever, rash, lymphadenopathy, and palmoplantar erythema. Coronary arteries become affected in up to a quarter of untreated patients, and this can lead to myocardial ischaemia and infarction.

\section{Small vessel vasculitis}

Small vessel vasculitis (leucocytoclastic or hypersensitivity vasculitis) is usually confined to the skin but may be part of a systemic illness. The rash is purpuric and sometimes palpable and occurs in dependent areas. The lesions may become bullous and ulcerate. Nailfold infarcts occur. Biopsies show a cellular infiltrate of small vessels often with leucocytoclasis (fragmented polymorphonuclear cells and nuclear dust). There are several causes of small vessel vasculitis, drugs and infection being the most common.
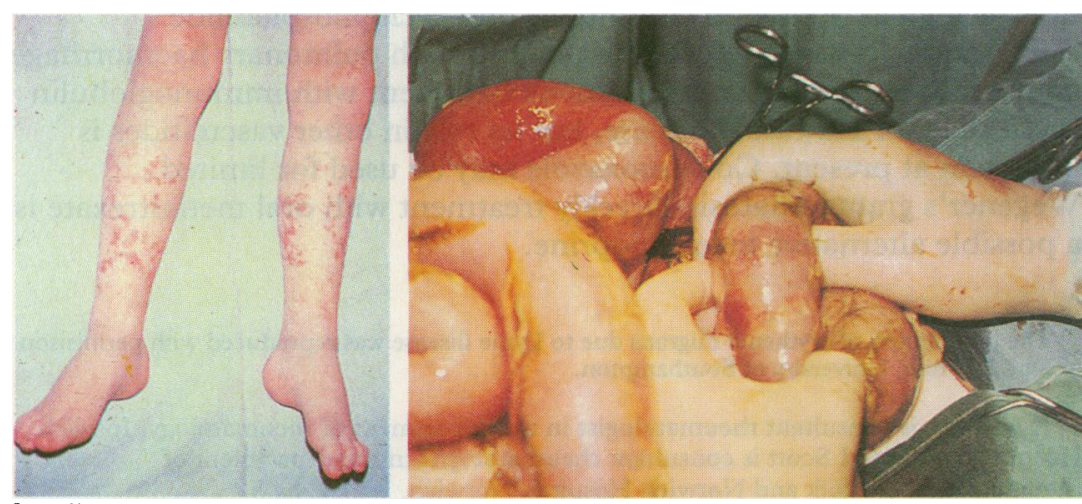

Small vessel vasculitis in Henoch-Schönlein purpura: (left) affecting the skin; (right) affecting the gut.
Henoch-Schönlein purpura is a form of small vessel vasculitis that occurs mainly in children and young adults. Patients present with rash, arthritis, abdominal pain, and sometimes renal impairment. Deposits of IgA can be detected in the skin and renal mesangium.

\section{Large vessel vasculitis}

This group of diseases includes giant cell arteritis and Takayasu's arteritis. The second condition is uncommon and affects young adults, who present with a non-specific illness and later with loss of pulses and claudication, especially of the upper limbs. 


\section{Investigation of vasculitis}

Assessing inflammation

- Urine analysis (proteinuria, haematuria, casts)

- Renal function tests (creatinine clearance,

24 hour protein excretion)

- Blood count (total, white blood cells,

eosinophils)

- Acute phase response (erythrocyte

sedimentation rate, $C$ reactive protein)

- Liver function tests

Immunological tests

- Autoantibodies (rheumatoid factor, antinuclear antibodies, antineutrophil cytoplasmic antibodies, anticardiolipin antibodies)

- Complement and immune complexes

- Cryoglobulins

Differential diagnosis

- Blood cultures

- Viral serology (hepatitis B, cytomegalovirus)

- Echocardiography

Specific investigations

- Radiographs of chest and sinus

- Biopsy of affected organs (especially

kidney)

- Angiography

\section{Investigation of vasculitis}

Investigation is directed towards establishing the diagnosis, the organs affected, and disease activity.

Urine analysis is the most important investigation since prognosis is determined mainly by the extent of renal impairment. Detection of proteinuria or haematuria in a patient with systemic illness requires immediate further investigation and is a medical emergency.

Blood tests-Leucocytosis suggests either primary vasculitis or infection, while leucopenia is associated with vasculitis secondary to a connective tissue disease. Eosinophilia suggests ChurgStrauss syndrome or a drug reaction.

Liver function tests-Abnormal results suggest either viral infection (hepatitis A, B, or C) or may be non-specific.

Immunology - Rheumatoid factors and antinuclear antibodies may indicate vasculitis associated with connective tissue disease. Measurement of antineutrophil cytoplasmic antibodies by indirect immunofluorescence is now widely available: cytoplasmic antineutrophil cytoplasmic antibodies are strongly associated with Wegener's granulomatosis, while perinuclear antineutrophil cytoplasmic antibodies are less so. Complement concentrations are low in infection and systematic lupus erythematosus but high in primary vasculitis.

Other investigations - Aneurysms can be revealed by angiography. Blood cultures, viral serology, and echocardiography are important to exclude other conditions that may present as severe multisystem disease and hence mimic vasculitis.

\section{Treatment and prognosis}

Treatment depends on the size of vessel affected. Small vessel vasculitis without necrotising features has an excellent prognosis and can usually be treated conservatively; patients with systemic disease respond well to a short course ( $<3$ months) of treatment with corticosteroids (20-60 mg oral prednisolone). Large vessel vasculitis also has a good prognosis and responds well to corticosteroids (40-60 mg oral prednisolone), but treatment is usually needed for more than a year. The dose of corticosteroid should be rapidly reduced according to clinical and laboratory parameters, with the aim of reducing the dose to $10 \mathrm{mg}$ or less within six months.

The introduction of cyclophosphamide has dramatically improved the prognosis of systemic necrotising vasculitis. Cyclophosphamide can be given either as continuous low dose oral treatment or as intermittent pulse therapy. The second method is preferred because of lower toxicity with intravenous therapy: cyclophosphamide's main toxic effects are haemorrhagic cystitis and formation of bladder tumours, and pulse therapy is significantly less toxic to the bladder than continuous oral therapy. Mesna may reduce this toxicity. Fertile men should be offered sperm storage before they are given treatment; ovarian function is less severely affected with pulse regimens.

Treatment with cyclophosphamide is continued for at least six months after remission before it is replaced by oral treatment with azathioprine. Survival has improved, but many patients require prolonged treatment (5-10 years) and there is still a substantial relapse rate, as high as $50 \%$ with continuous oral cyclophosphamide.

Plasmapheresis is reserved for patients with pulmonary haemorrhage and severe renal disease. Intravenous treatment with immunoglobulin is effective for Kawasaki disease, but its role in other vasculitides is uncertain at present. Co-trimoxazole may be used for limited Wegener's granulomatosis. Weekly treatment with oral methotrexate is a possible alternative to azathioprine.

The photograph of erythema migrans due to Lyme disease was reproduced with permisison of Dr J E White, University of Southampton.

R A Watts is consultant rheumatologist in the department of rheumatology, Ipswich Hospital, and D G I Scott is consultant rheumatologist in the department of rheumatology, Norfolk and Norwich Hospital, Norwich.

The ABC of Rheumatology is edited by Michael L Snaith, senior lecturer in rheumatology at the Institute for Bone and Joint Medicine, University of Sheffield. 